\title{
The Impact of Emerging Software Paradigms on Software Quality and User Expectations
}

\author{
Dennis B. Smith \\ Carnegie Mellon University/ Software Engineering Institute \\ United States \\ dbs@sei . cmu .edu
}

This talk will discuss emerging approaches to software development and evolution that enable organizations to respond quickly to new business needs while maintaining their legacy applications. These approaches include:

- Service oriented architecture (SOA) which is a way of designing, developing, deploying, and managing systems where coarse-grained services represent reusable functionality, and service consumers compose applications or systems using the functionality provided by these services through standard interfaces. This approach enables the flexible composition and evolution of new services. Major barriers include lack of a long term strategy, lack of effective governance, unrealistic expectations, and inappropriate technical strategy.

- Cloud computing which is a "a large-scale distributed computing paradigm that is driven by economies of scale, in which a pool of abstracted, virtualized, dynamically-scalable, managed computing power, storage, platforms, and services are delivered on demand to external customers over the Internet [1]. This approach enables consumer organizations to have access to state of the practice hardware and software without making many of the upfront infrastructure investments. The main drivers for cloud computing adoption include scalability, elasticity, virtualization, cost, mobility, collaboration, and risk reduction. Major barriers include security, interoperability, control and performance.

- Enterprise architecture planning and development which develops a comprehensive plan for using business functionality across an enterprise and building applications from shared resources. Because it takes a perspective that crosses an entire enterprise, it enables the breaking down of barriers that take place within individual organizations. Major barriers include lack of long term commitment, and a focus on completeness rather than practicality.

The combination of these three approaches offers potentials for both success and failure. They can enable rapid responses to new business situations through the shared use of common resources, as well as the discipline to use a common plan for implementing enterprise wide priorities. However, the use of these approaches has often been over-hyped, resulting in failure. This talk uses lessons learned from current adoption efforts to identify the core concepts of these approaches, what their potentials are, as well as common misconceptions and risks.

\section{Reference}

1. Foster, I., Zhau, Y., Ioan, R., Lu, S.: Cloud Computing and Grid Computing: 360-Degree Compared. In: Grid Computing Environments Workshop (2008) 